\title{
CONSTRUCCIONES NORMALIZADAS. SISTEMA ESTRUCTURAL DE MADERA PARA VIVIENDAS UNI Y BIFAMILIARES
}

\author{
(STANDARDIZED CONSTRUCTIONS. STRUCTURAL WOOD SYSTEM FOR DETACHED \\ AND SEMIDETACHED HOUSES)
}

Ricardo Novaro Bocco y Daniel Manso Texo, Arquitectos Centro de Investigación del Medio Ambiente y el Desarrollo (CIMADe) ESPAÑA

\section{RESUMEN}

Un aporte tecnológico para la normalización de la construcción

Mediante una sistemática del diseño arquitectónico y el uso de la madera como elemento estructural, se ha obtenido un sistema constructivo que permite la incorporación de los materiales que ofrece el mercado por elemento y por componentes.

Las principales características del sistema constructivo son el invertir el orden de dos de los procesos constructivos tradicionales, realizando primero la cubierta (arriba-abajo) y la envolvente interior (dentro-fuera).

El elemento estructural de madera está combinado con piezas metálicas que mejoran su trabajo resistente y optimizan el proceso constructivo.

Las ventajas obtenidas por este procedimiento con la utilización de la madera son:

- Diseño y elementos constructivos estandarizados, normalizados y modulados.

- Técnicas constructivas sencillas, con reducción en los tiempos de construcción.

- Soluciones formales y materiales flexibles y adaptables a cada región.

- Rendimiento óptimo de materiales y mano de obra.

- Mejora en la relación Precio-Calidad.

El artículo que a continuación se desarrolla presenta los resultados obtenidos en la investigación y desarrollo Ilevados a cabo por el Departamento de Tecnología del Centro de Investigación del Medio Ambiente y el Desarrollo (CIMADe).

\section{SUMMARY}

A technological contribution for the construction normalization

By means of a systematic for the architectural design and the use of wood as an structural element, it is been

obtained a constructive system wich allows the incorporation of the materials wich the market offers by elements and components.

The main traits of the constructive system are the possibilities of reversing the order of two of the traditional constructive process, first by putting into effect the cover (up-down) and the interior surround (inside-outside).

The structural element of wood is combined with metallic pieces wich improve it's resistance work and optimizes the constructive process.

The advantage obtained in this process by using wood are:

- Design and constructive element standardized, normalized and modulated.

- Simple constructive techniques with less construction time.

- Formal solutions and flexible materials, adaptable to each region.

- Optimum output of materials and labour.

- Improve the relation Quality-Price.

The article developed shows the results obtained in the investment and performance carried out by the Technological Department of the Investment Center of Environment and Development. 


\section{INTRODUCCIÓN}

\section{La madera como material de la construcción}

La madera como material de construcción y la tecnología que la desarrolló históricamente han ido de la mano, ello se puede apreciar en un simple repaso retrospectivo de la arquitectura e ingeniería civil, naval y militar.

Dicha tecnología ha sido un factor determinante para el mayor o menor empleo de la madera en la construcción, principalmente en su faz estructural o resistente.

El hierro, el hormigón y otros materiales (desarrollados significativamente en los dos últimos siglos) habian relegado a la madera como sistema estructural en los inicios del siglo XX, sin embargo la aparición de nuevos materiales y técnicas de elaboración han permitido relanzar a los primeros planos a la madera en la construcción.

La madera aserrada que se usaba tradicionalmente, para trabajos resistentes, tendió a desaparecer por las limitaciones que ofrecía en el tamaño de las luces y los requerimientos estructurales.

Las maderas laminadas encoladas, los tableros aglomerados y contrachapados y los productos químicos para la protección contra el fuego, la humedad y los insectos, han sido entre otros los factores determinantes para colocar a la madera como un material altamente competitivo frente a otros como el acero o el hormigón.

\section{Características generales}

Las principales características de la madera como material de la construcción se pueden agrupar en los siguientes puntos:

- Buena resistencia a la flexión en la relación peso resistencia.

- Buena resistencia a la tracción y a la compresión.

- Rotura progresiva y no instantánea.

- Aceptable aislamiento térmico y acústico.

- Estabilidad ante la acción del fuego.

- Bajo módulo de elasticidad longitudinal.

- Mínima resistencia a las tensiones tangenciales horizontales (baja resistencia a los esfuerzos de cortes). Esto limita las posibilidades de generar secciones aligeradas en forma de T, doble T o en cajón.

- Óptima combinación de madera aserrada con tableros que permite múltiples soluciones como forjados mixtos más livianos y resistentes, eliminando la falta de monolitismo, el exceso de deformación por falta de rigidez y la poca economía a partir de luces de 4 ó 5 metros de los forjados construidos exclusivamente con madera aserrada.

- Limitación considerable de las secciones de madera natural y aserrada que admiten pocas dimensiones en sus luces.

- Dependencia en las uniones con otros materiales como el acero que funcionan mejor que las exclusivamente en madera.

- Un menor porcentaje de humedad le confiere al material mayor resistencia, pero reduce su capacidad a la rotura.

La inercia a la agresión de los agentes externos es una cuestión a considerar con relativa importancia sobre todo en lo que respecta a los hongos y a los insectos.

\section{La madera dentro de un proceso industrial}

La industrialización de un sistema constructivo independientemente del material que se utilice ofrece en principio un abanico más amplio de productos técnicamente controlados y por ende con mayor calidad en la elaboración.

Es un hecho comprobado que la producción industrial de sistemas o componentes constructivos no conlleva necesariamente una repetición despersonalizadora de los edificios, sino por el contrario una rica y mayor libertad en las formas estéticas que impiden una masificación y monotonía en el producto, claro está siempre y cuando existan en su concepción ideas imaginativas, de lo cual no se eximen tampoco las soluciones artesanales mal diseñadas.

La madera como material industrializado para la construcción, comparado con respecto a sus principales competidores el hierro, el acero y el hormigón armado, ofrece un conjunto de ventajas a tener en cuenta, como que:

- La madera es un material renovable (estimándose de manera aproximada 20 a 25 años para las coníferas y 100 a 150 años para las frondosas).

- La obtención del material no requiere ningún tipo de energía artificial ya que el árbol se genera en base a energías naturales.

- La elaboración industrial no demanda altos costos energéticos y su proceso es ágil, relativamente fácil y no es contaminante.

- La tecnología industrial de elaboración del material permite obtener un producto preciso y con sello de calidad.

- La tecnología que se emplea en el tratamiento para el secado y la protección del material es fundamen- 


\section{LAS CONSTRUCCIONES NORMALIZADAS}

tal para el buen rendimiento posterior de los componentes.

- Los tipos de sistemas constructivos pueden ser en base a madera sólida, laminada y de tableros.

- La efectividad de la producción industrial está condicionada por la adecuada coordinación modular y dimensional de los sistemas estructurales, de los componentes prefabricados y de los elementos de acabados.

- Los sistemas constructivos de viviendas no requieren en demasía mano de obra especializada, ni maquinaria adicional para su montaje.

- La construcción por componentes permite una mayor oferta de soluciones estéticas y constructivas.

\section{Panorama europeo y español}

En la Europa occidental en las últimas décadas el empleo de la madera como material de la construcción, particularmente en el mercado de las viviendas uni y bifamiliares, ha experimentado un constante crecimiento en valores tanto absolutos como relativos.

Los factores que han influido para que se produzca este fenómeno han sido entre otros el retorno a la vivienda individual, el resurgir de las actuaciones residenciales de pequeño y mediano tamaño, la necesidad de disminuir los costos energéticos en la producción y posterior mantenimiento de las construcciones, el menor coste de la construcción de la vivienda de madera con respecto a la de otros materiales, la diversidad de componentes prefabricados y la menor demanda de mano de obra especializada que demanda la vivienda en madera para su construcción.

Estos factores han permitido que en los países nórdicos se llegue a superar el 85 y $90 \%$ del total de viviendas unifamiliares construidas, con el sistema estructural de madera.

La madera tiene en España cierta tradición constructiva a nivel de construcciones artesanales, sin embargo a nivel industrial los antecedentes son casi nulos.

Ello se ve reflejado en la ausencia de un sólido y auténtico cuerpo de normativas sobre cálculo, fabricación y montaje de elementos resistente de la madera.

En España se requiere durante los próximos años una actuación integral a todos los niveles institucionales, para que se incorpore de manera efectiva la madera como material industrializado de la construcción, particularmente en lo que se refiere al trabajo estructural de dicho material.

\section{Presentación}

Las CONSTRUCCIONES NORMALIZADAS pertenecen al programa de INVESTIGACIÓN TECNOLÓGICA PARA LA VIVIENDA (ITV) que se desarrolla en el área de Tecnología del Centro de Investigación del Medio Ambiente y el Desarrollo (CIMADe) de Madrid.

El programa pertenece al Tipo I+D (investigación más desarrollo) y se compone de varias áreas de investigación preferentes.

En el área preferente denominada Sistema Constructivo Inverso (SCl) se ha desarrollado un Sistema Estructural mixto, compuesto básicamente de madera con el apoyo específico de piezas metálicas. La tipologia de madera también es del tipo mixto ya que está compuesta por elementos puntuales y elementos de paneles.

El sistema que actualmente sale al mercado es fruto de varios años de investigación y desarrollo de diversas generaciones de prototipos, ensayados, mejorados y puesto a punto en la elaboración de sus componentes y en el sistema de montaje.

\section{Características principales del sistema}

El Sistema Constructivo Inverso consiste en invertir el orden de dos de los procesos constructivos tradicionales, realizando primero la cubierta: arriba - abajo y la envolvente interior dentro - fuera (Fig. 1).

Las ventajas obtenidas por este procedimiento y con la utilización de la madera son:

- Elementos constructivos estandarizados, normalizados y modulados - con propiedades aislantes y estructurales óptimas-.

- Técnicas constructivas sencillas - con mano de obra especializada casi nula y sin maquinaria de montaje-.

- Diseño racionalizado, flexible y abierto con soluciones distintas y personalizadas - con adaptaciones estéticas y formales-.

- Recintos ampliables y modificables - con una amplia variedad en los programas funcionales y de superficies- (Fig. 2).

- Materiales de la construcción adaptados a cada región - con una aceptable resistencia a los efectos sísmicos y a las condiciones climáticas-.

- Rendimiento óptimo de los materiales y de la mano de obra - con una aligeración en el peso de la construcción y una reducción en las dimensiones de las cimentaciones y de las superficies de los espacios no habitables como los muros-.

http://informesdelaconstruccion.revistas.csic.es 


\section{Elementos constructivos}

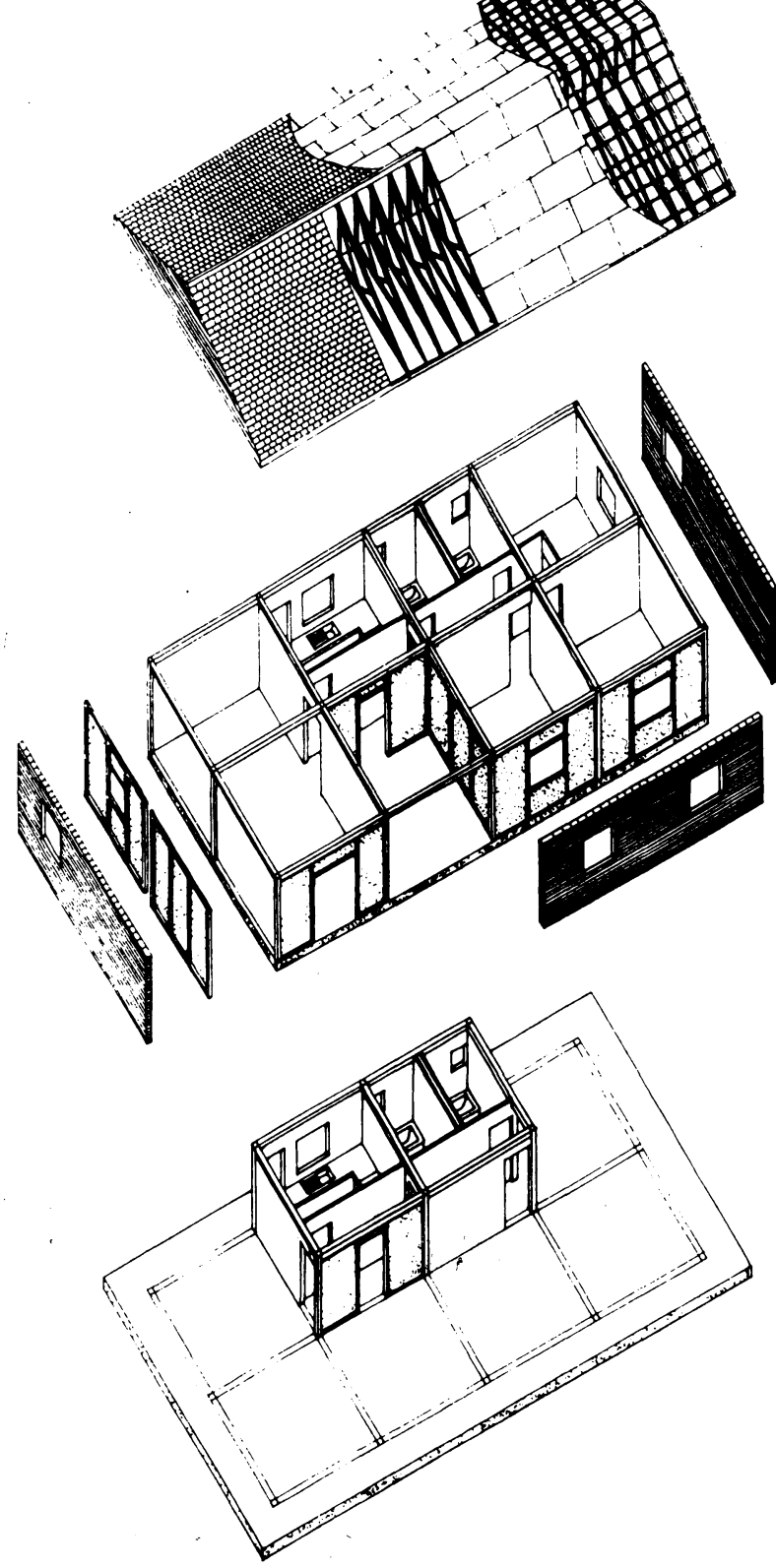

Fig. 1

- Mejora de los niveles de habitabilidad - con un mayor aislamiento térmico acústico, eliminación de puentes térmicos y una mejor regulación de la humedad-.

- Concentración de la tecnología compleja de las instalaciones - con una colocación en seco y en tiem. pos mínimos-.

- Mejora en la relación precio calidad - con la reducción en los tiempos de construcción se reducen los costos financieros y los acabados industriales permiten un trabajo en obra más limpio y fácil. También a igual monto de inversión se puede obtener mayor número de viviendas-.

\section{EL PROCESO CONSTRUCTIVO}

(Fig. 3 y fotos 1,2 y 3 )

El proceso constructivo del SCl consta de las siguientes etapas:

\section{Soporte:}

1) Construcción de un plano horizontal que recibirá al edificio y lo vinculará con el terreno. Las posibilidades de este plano son desde una solera apoyada directamente sobre el terreno o un forjado de madera o un forjado de hormigón apoyados sobre pilotes o cimientos corrido de un pie de ladrillo macizo. Aislamiento. Es fundamental la nivelación de esta superficie para lograr la máxima exactitud del siguiente paso.

2) Realización de un replanteo (ajustado y muy exacto) mediante guías especialmente diseñadas para ello.

3) Colocación y fijación de casquillos metálicos.

4) Colocación de pilares de madera con casquillos metálicos superiores incorporados y recibido de vigas de madera de carga y atado.

5) Colocación de guías de replanteo para cerchas.

6) Colocación de cerchas de madera con conectores.

7) Fijación de rastreles o de paneles con rastreles. Aislamiento.

Envolvente superior exterior $y$ envolvente interior:

1) Colocación en seco de las tejas, chapas u otro material destinado a la cubierta.

2) Fijación de los bastidores de madera perimetrales.

3) Colocación de paneles de cartón yeso o de yeso o de madera interior en paredes y falsos techos.

4) Instalación del MTS.

5) Colocación de solado (opcional).

6) Colocación y fijación de las instalaciones (eléctrica, calefacción, agua, TV, etc).

7) Acabados interiores.

\section{Envolvente exterior:}

1) Se construye de manera tradicional o industrializando los muros exteriores con materiales que se adecúen a las condiciones geográficas, culturales, climáticas, etc., del lugar.

http://informesdelaconstruccion.revistas.csic.es 
PLANTAS

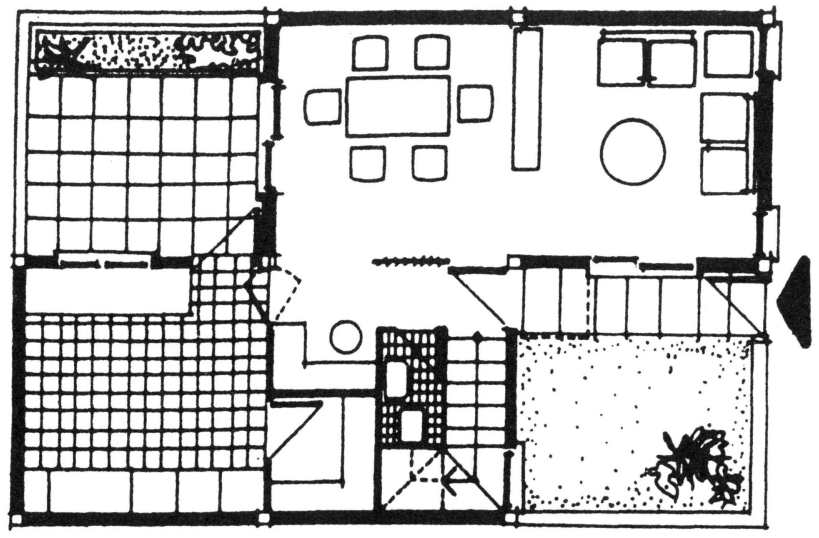

Tipo 8 M (Sup.: $112 \mathrm{~m}^{2}$ )

planta baja

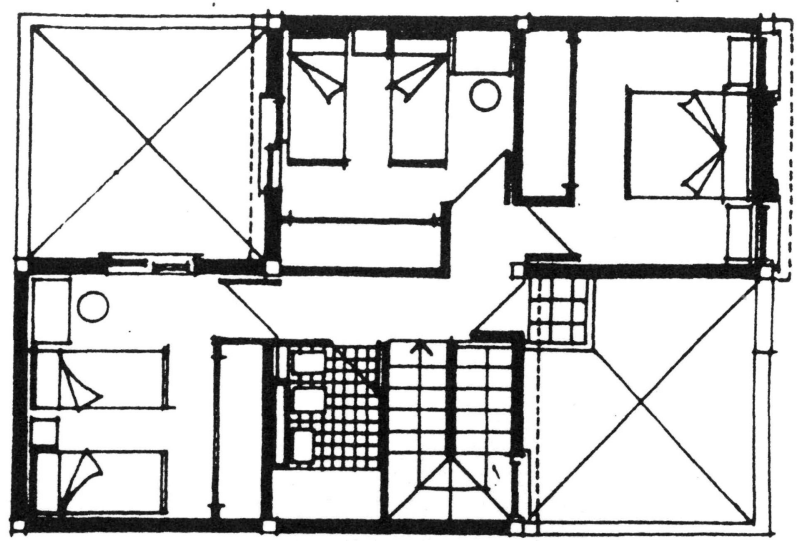

planta alta (3 dorm.)

Tipo 12 M (Sup.: $168 \mathrm{~m}^{2}$ )

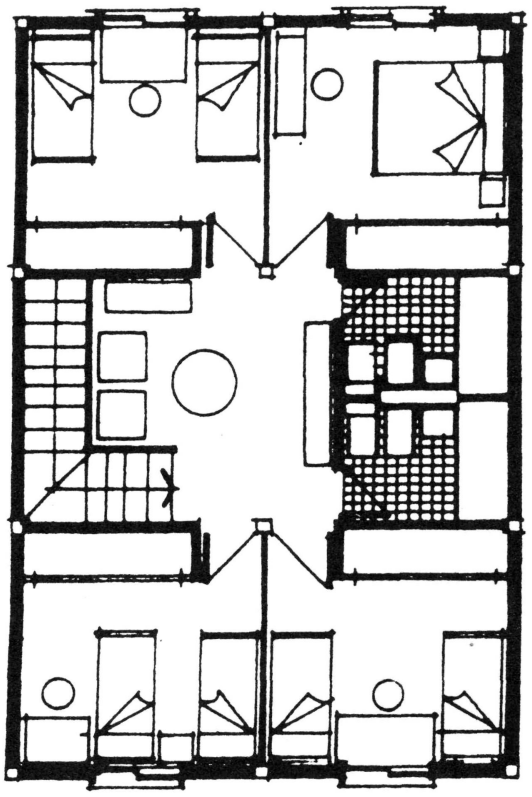

planta alta (4 dorm.)

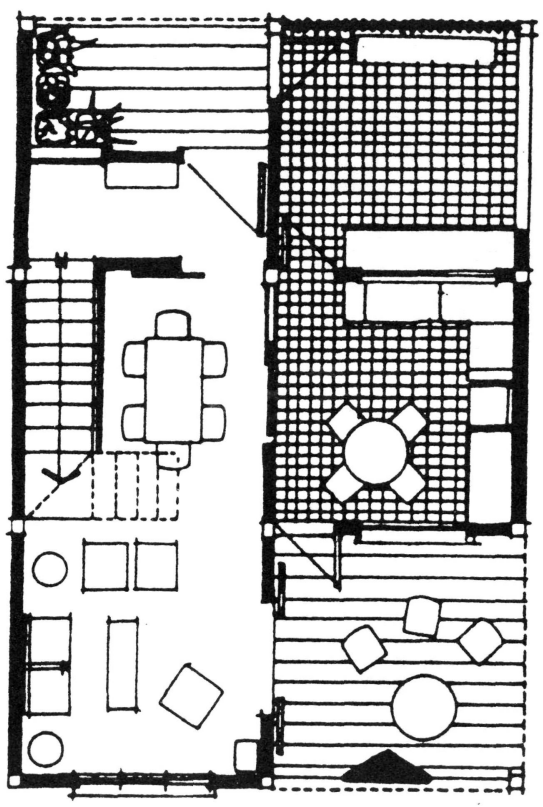

Tipo 8 M (Sup.: $112 \mathrm{~m}^{2}$ )

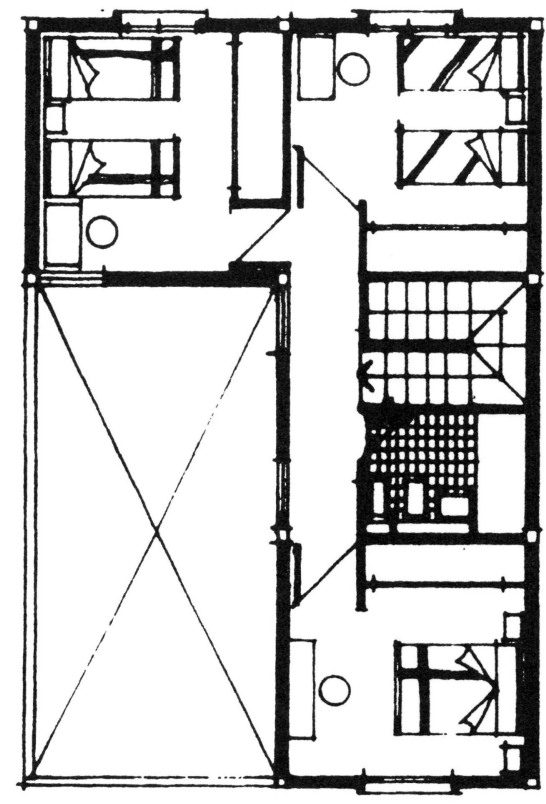

planta alta (3 dorm.)
Tipo 9 M (Sup.: $\left.126 \mathrm{~m}^{2}\right)$

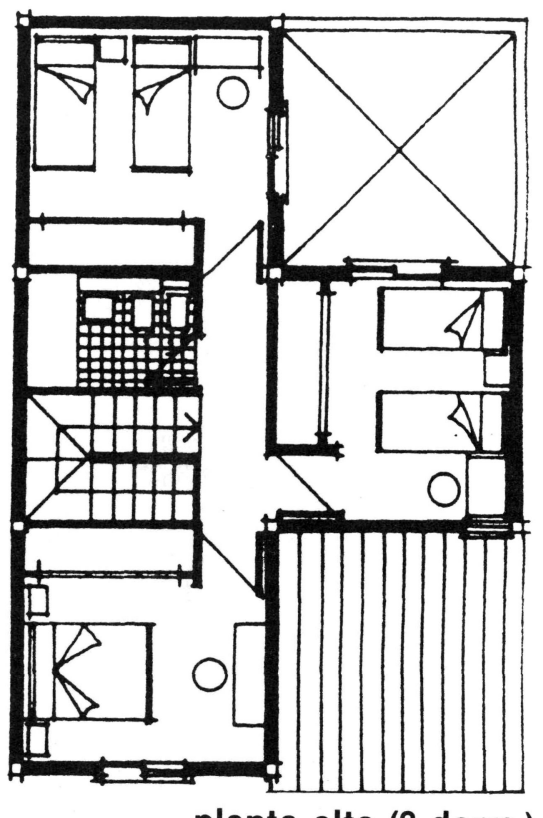

planta alta (3 dorm.)
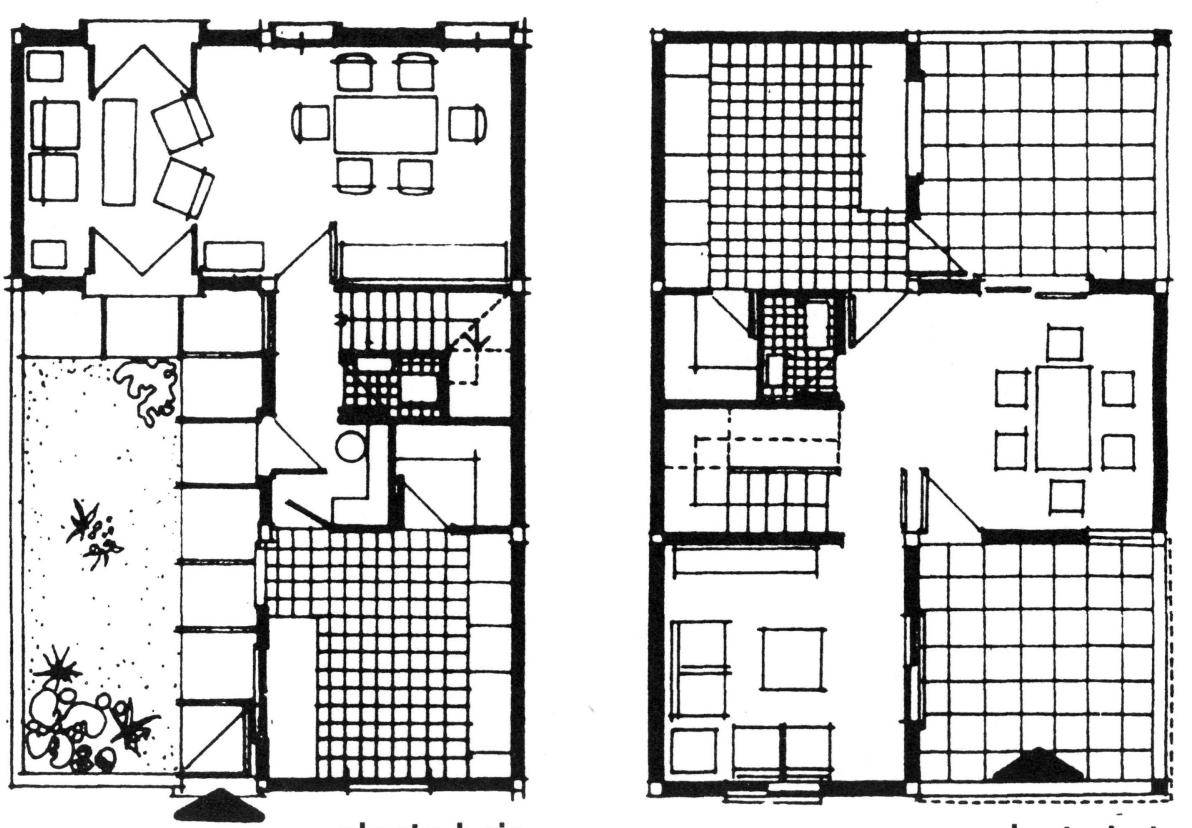

planta baja $_{\text {http://informesdelaconstruccion.revislas.csic.es baja }}$ 


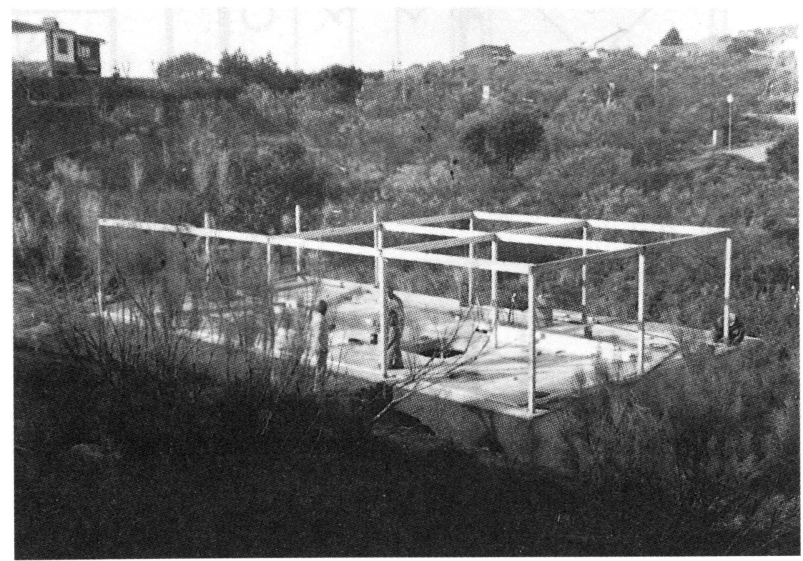

Foto 1

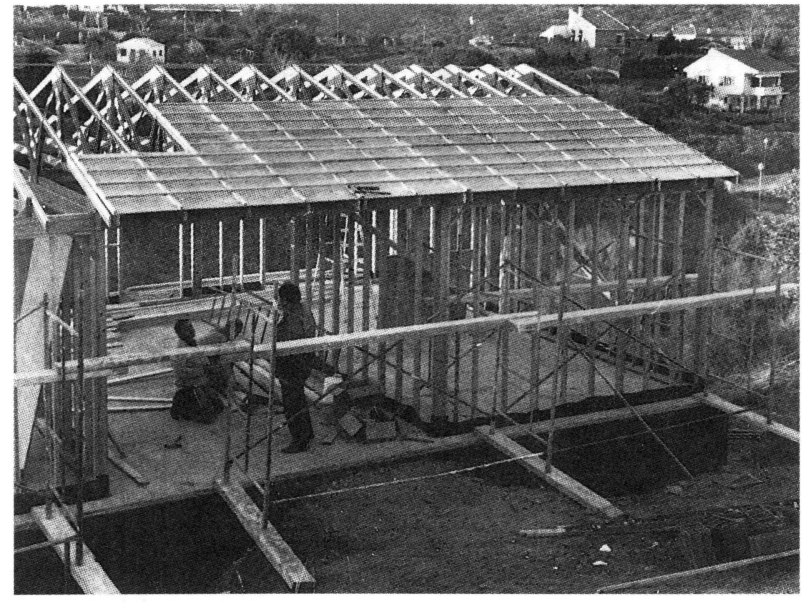

Foto 2

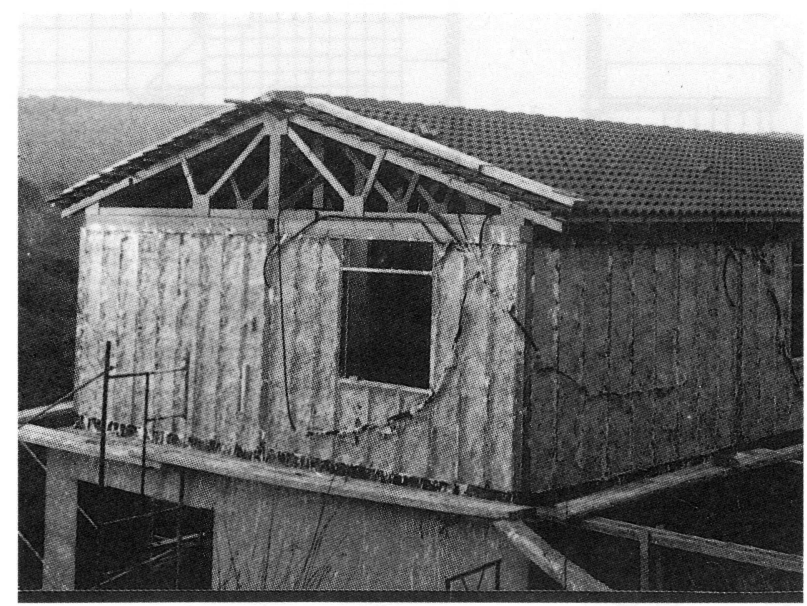

Foto 3

(c) Consejo Superior de Investigaciones Científicas Licencia Creative Commons 3.0 España (by-nc)

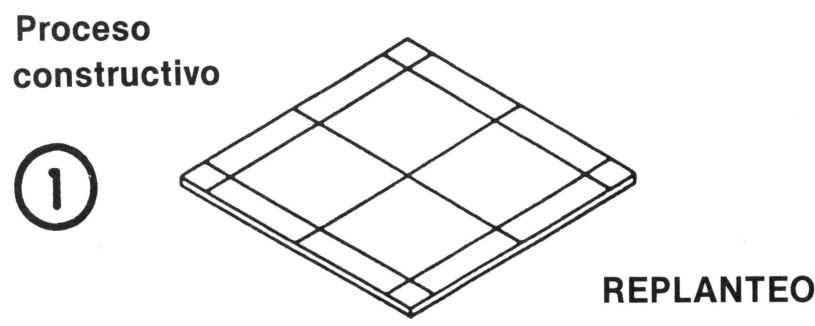

(2)

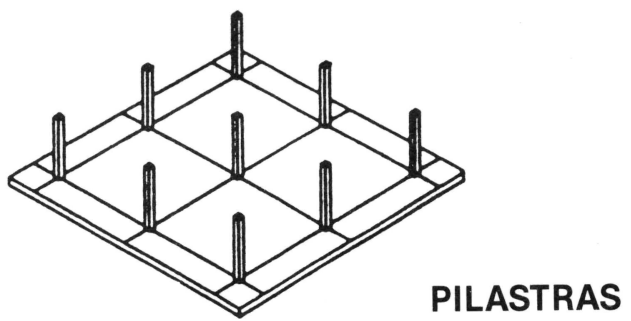

(3)

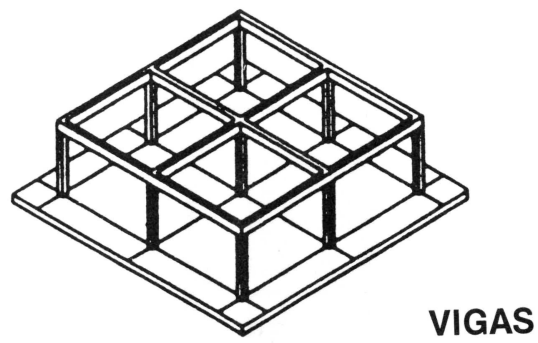

(4)

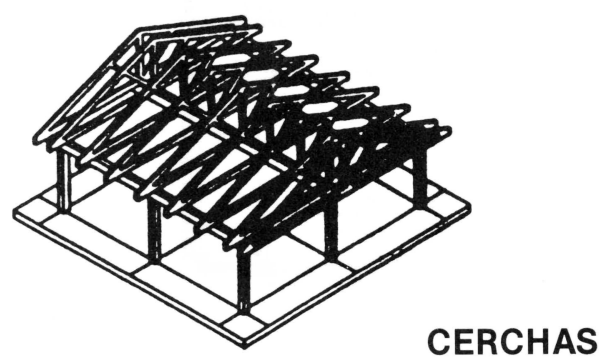

(5)

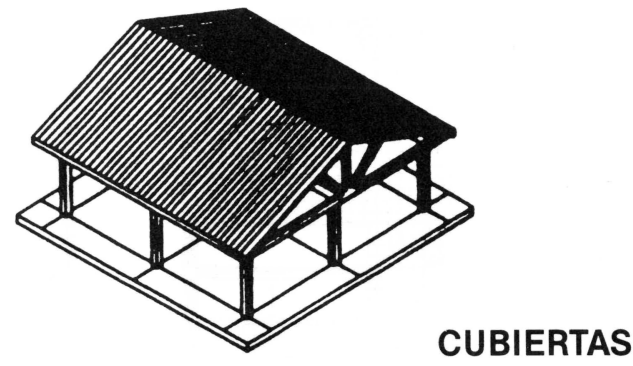

(6)

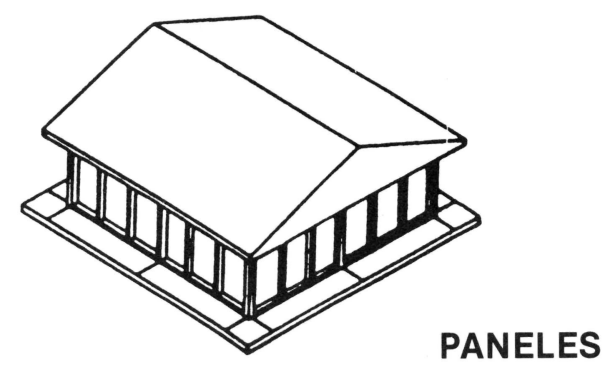

Fig. 3

http://informesdelaconstruccion.revistas.csic.es 


\section{TIPOLOGÍAS CONSTRUCTIVAS Y FUNCIONALES}

Las C.N se desarrollan en base a las siguientes tipologías constructivas:

- Emergencia.

- Autoconstruida.

- Desmontable.

- Permanente.

Las tipologías funcionales son:

- Viviendas.

- Escuelas.

- Asistenciales.

- Albergues.

\section{COMPONENTES FIJOS Y VARIABLES DEL SISTEMA} (Fig. 4)

El sistema se compone de elementos fijos y elementos variables. Los componentes fijos corresponden al soporte o estructura y los componentes variables a los cerramientos o envolvente:

\section{- Soporte de Madera:}

- Pilares de madera maciza.

- Vigas de atado y distribución de madera.

- Bastidor de madera autoportante.

- Cerchas de conectores:

- Soporte de metal.

- Casquillos de fijación (sup. e inf.).

- Conectores de grapas de chapa galvanizada.

Los elementos constructivos variables son:

- Cerramiento horizontal:

- Solera.

- Forjado de madera sobre pilotes.

- Forjado de hormigón.

- Cerramiento interior vertical y horizontal:

- Madera.

- Cartón yeso.

- Yeso.

- Otros.

- Cerramiento vertical exterior:

- Madera.

- Ladrillo.

- Piedra.

- Metálico.

- Otros.

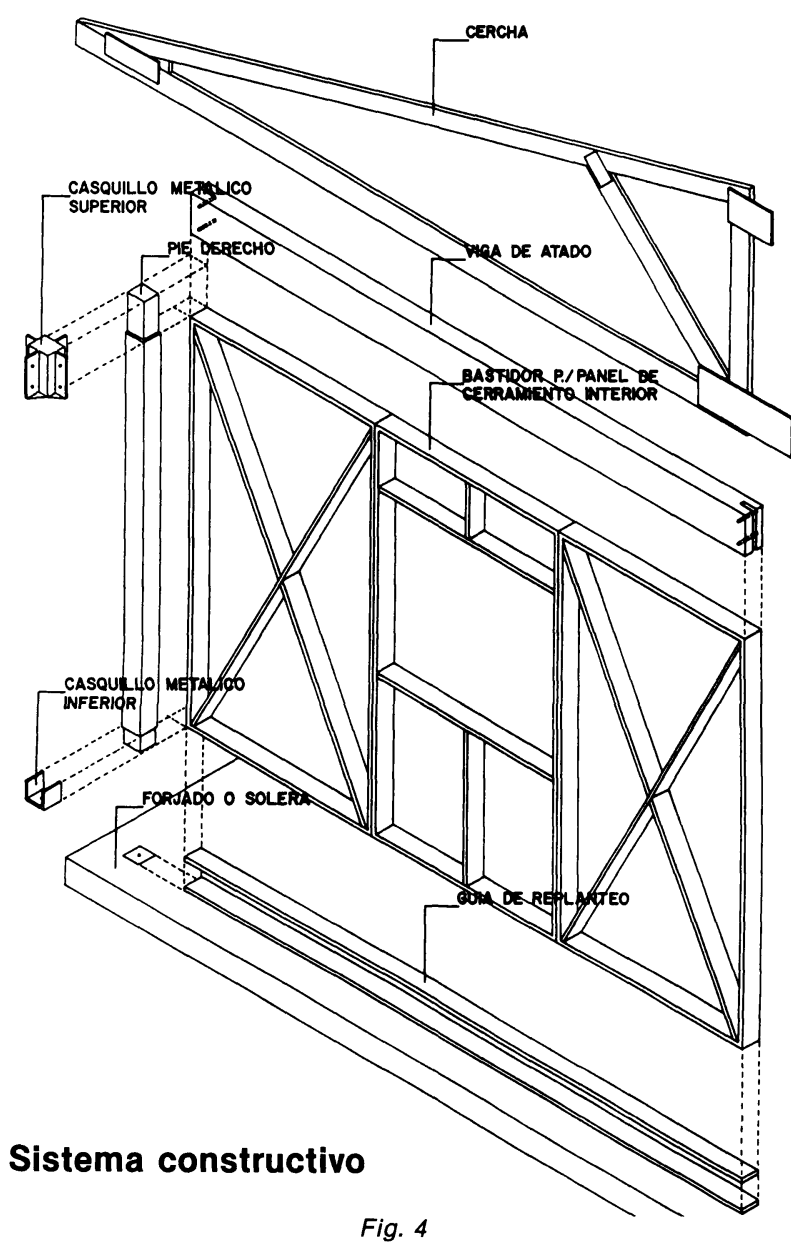

- Cerramiento horizontal exterior:

- Tejas cerámicas.

- Tejas de hormigón.

- Chapas galvanizadas.

- Otros.

\section{BIBLIOGRAFÍA}

- C.M.H.C.

CANADIAN WOOD FRAME - HOUSE CONSTRUCTION METRIC EDITION - CANADA 1979

- C.F.I.B.C. TIMER FRAME CONSTRUCTION CANADA 1978

- K. HOFFMAN Y H. GRIESE CONSTRUCCIONES EN MADERA E. BLUME, BARCELONA 1967

- O.D.IT.A.

EL TABLERO AGLOMERADO EN LA CONSTRUCCIÓN MADRID 1979

- I.N.I.A. - A.N.C.O.P. I. II. Y III. JORNADAS NACIONALES DE LA MADERA EN CONSTRUCCIÓN. MADRID 1984/85/86

- AMERICAN PLYWOOD ASSOCIATION PUBLICACIONES VARIAS $1983 / 89$

- CONSEJO SUECO DE INFORMACIÓN DE LA MADERA PUBLICACIONES VARIAS: TRÄINFORMATION ESTOCOLMO 195/89. 